\title{
EVALUATION OF PREANALYTICAL ERRORS IN CLINICAL BIOCHEMISTRY LABORATORY IN A TERTIARY CARE HOSPITAL IN KOLKATA
}

\author{
Sayari Banerjee ${ }^{1}$, Roy Choudhury $J^{2}$, Indranil Chakraborty 3 \\ 1Demonstrator, Department of Biochemistry, College of Medicine and Sagore Dutta Hospital. \\ 2Demonstrator, Department of Biochemistry, College of Medicine and Sagore Dutta Hospital. \\ 3 Professor and HOD, Department of Biochemistry, College of Medicine and Sagore Dutta Hospital.
}

\section{ABSTRACT}

\section{BACKGROUND}

Accurate laboratory results are vital for patients' safety. Errors in laboratory may occur in the pre-analytical, analytical and post analytical phase. In the present study, we tried to identify the types of the pre-analytical errors in clinical biochemistry laboratory in a tertiary care hospital in Kolkata by retrospective analysis.

\section{MATERIALS AND METHODS}

This study is a retrospective descriptive study. 164, 946 test requests were received, different types of errors were registered by the laboratory personnel. Percentage of occurrence of errors, defects per million (DPM) and Sigma value of each error were calculated.

\section{RESULTS}

$99.97 \%$ of the requisitions missed clinical information. 7.7\%, 7\% and 6.2\% (sigma metric 3, 3 and 3.1) samples were without proper mention of sample collection dates, sex and age of the patient respectively. Among errors in the sample, percentage of haemolysed samples were the highest (5.2\% with sigma metric 3.2$)$ followed by insufficient sample quantity (1.6\% with sigma metric 3.7) and clotted sample (1.5\% with sigma metric 3.7$)$.

\section{CONCLUSION}

Proper awareness in preanalytical procedure is need of the hour. Training of personnel responsible for preparation of patient, collection of biological sample and transportation of samples should be done with utmost care, so that pre-analytical error can be minimized.

\section{KEYWORDS}

Pre-Analytical Error, Error Percentage, Sigma Value.

HOW TO CITE THIS ARTICLE: Banerjee S, Roy Choudhury J, Chakraborty I. Evaluation of preanalytical errors in clinical biochemistry laboratory in a tertiary care hospital in Kolkata. J. Evolution Med. Dent. Sci. 2018;7(09):1118-1120, D0I $10.14260 /$ jemds/2018/254

\section{BACKGROUND}

Laboratory investigations have a great impact in diagnosis and treatment of patients in present time. So, accurate laboratory results are vital for patient safety. Clinical laboratory errors directly lead to inappropriate clinical decisions as well as sample rejection and hence increased turnaround time culminating in delayed diagnosis and longer hospital stays which ultimately end up in added cost to laboratory and increased healthcare costs, decreased patient and physician satisfaction.

In the year 1981, Lundberg introduced the concept of the 'brain-to-brain loop' for describing the total testing process (TTP), which includes nine steps starting from ordering, collection, identification, transportation, separation or preparation, analysis, reporting, and upto action. ${ }^{1}$ The total testing process generally comprises three phases-1) Events before the sample test/analysis (Pre-analytical

'Financial or Other Competing Interest': None.

Submission 19-01-2018, Peer Review 14-02-2018,

Acceptance 20-02-2018, Published 26-02-2018.

Corresponding Author:

Dr. Jayati Roy Choudhury,

Department of Biochemistry,

College of Medicine and Sagore Dutta Hospital,

Kamarhati, Kolkata-700058.

E-mail: jayatijayati_rc@rediffmail.com

DOI: $10.14260 /$ jemds $/ 2018 / 254$

\section{(c) $(\mathbf{P}) \Theta$}

phase), 2) Analysis of sample (analytical phase) and 3) Events after the analysis (post analytical phase). Quality in laboratory medicine should be defined as the guarantee that each and every step in the total testing process (TTP) is correctly performed. ${ }^{2} \mathrm{~A}$ quality indicator is thus 'an objective measure that potentially evaluates all critical care domains as defined by the Institute of Medicine (IOM) (patient safety, effectiveness, equity, patient-centredness, timeliness and efficiency), that is based on evidence associated with those domains, and can be implemented in a consistent and comparable manner across settings and over time.' 3

Laboratory errors may be defined as "any defect from ordering tests to reporting results and appropriately interpreting and reacting on these." 4 Despite advanced automation in diagnostic labs, there are still considerable error rates at clinical diagnostic labs. ${ }^{5}$ Previous studies have focused on the analytical phase of diagnostic tests, and many quality control programs were initiated at diagnostic labs to monitor analytical phase errors. However, pre- and postanalytical errors were neglected worldwide. Interestingly, a majority of diagnostic lab errors are either pre-analytical (46-68\%) or post-analytical (18-47\%). Indeed, only 7-13\% of errors actually occur during the analytical phase. ${ }^{6}$ In this background, we tried to identify the types of the preanalytical errors in clinical biochemistry laboratory in a tertiary care hospital in Kolkata with a goal to minimize the pre-analytical error by improving the knowledge, attitude 
and skill of persons involved in preparation of patient and collection and transport of biological samples to laboratory.

\section{MATERIALS AND METHODS}

This study, a retrospective descriptive study was done after approval of the study proposal by the institutional ethics committee. A retrospective analysis for errors in the preanalytical phase in the clinical chemistry laboratory in a tertiary care hospital in Kolkata has been carried out. Upon sample receiving, any defect in the specimen was visually detected by the investigator. Different types of errors, rejections and its causes were then registered by the laboratory personnel following the quality indicators developed by the International Federation of Clinical Chemistry and Laboratory Medicine Working Group for Laboratory Errors and Patient Safety (IFCC-WG-LEPS) for the pre-analytic phase. ${ }^{7}$ Percentage of occurrence of defects was calculated as-

(Number of defects $\div$ Total number of tests) X 100

Defects per million (DPM) and Sigma value of each error were calculated, using the Westgard calculator ${ }^{8}$. According to the table of equivalence between the Sigma level and defects per million (DPM), an error rate of $6.68 \%$ corresponds to a Sigma value 3 , equivalent to borderline unacceptable, while an error rate of $0.62 \%$ corresponds to a Sigma value 4 , which reflects a good level of control.8,9

\section{RESULTS}

164, 946 test requests were received during the data collection period, Errors were documented owing the following reasons: $99.97 \%$ of the requisitions missed clinical information (Sigma values were $<0.1$ ), in $7.7 \%$ of samples collection dates were missing. $7 \%$ and $6.2 \%$ samples were without proper mention of sex and age of the patient respectively. Sigma values were 3 in case of missed collection date and 3 and 3.1 in cases of absent or wrong information about patient's age and sex. While considering errors in sample, $5.2 \%$ samples (sigma value 3.2 ) were haemolysed and $1.6 \%$ (sigma value 3.7 ) had insufficient sample quantity and in $1.5 \%$ (sigma value 3.7 ) samples were clotted. No parameters could reach six sigma value.

\begin{tabular}{|c|c|c|c|}
\hline $\begin{array}{c}\text { Erroneous } \\
\text { Patient ID }\end{array}$ & $\begin{array}{c}\text { Avg.\% of } \\
\text { Error in One } \\
\text { Year Period }\end{array}$ & D P M & Sigma \\
\hline $\begin{array}{c}\text { Name (absent / } \\
\text { wrongly written) }\end{array}$ & 0.3 & 3092 & 4.3 \\
\hline $\begin{array}{c}\text { Age (absent / } \\
\text { wrongly written) }\end{array}$ & 6.2 & 622202 & 3.1 \\
\hline $\begin{array}{c}\text { Sex (absent / } \\
\text { wrongly written) }\end{array}$ & 7 & 70265 & 3 \\
\hline $\begin{array}{c}\text { Reg. no. (absent } \\
\text { /wrongly written) }\end{array}$ & 0.4 & 3880 & 4.2 \\
\hline $\begin{array}{c}\text { Ward/unit/physician } \\
\text { (absent /wrongly } \\
\text { written) }\end{array}$ & 0.3 & 2546 & 4.4 \\
\hline $\begin{array}{c}\text { Date (absent / } \\
\text { wrongly written) }\end{array}$ & 7.7 & 76995 & 3 \\
\hline $\begin{array}{c}\text { Clinical information } \\
\text { ( absent ) }\end{array}$ & 99.9 & 999679 & $\begin{array}{c}\text { Below the } \\
\text { lower limit } \\
\text { of } \\
\text { calculation }\end{array}$ \\
\hline \multicolumn{2}{|c|}{ Table 1 } & \multicolumn{2}{|c|}{} \\
\hline
\end{tabular}

\begin{tabular}{|c|c|c|c|}
\hline Errors in Sample & $\begin{array}{c}\text { Avg.\% of } \\
\text { Error in One } \\
\text { Year }\end{array}$ & D P M & Sigma \\
\hline $\begin{array}{c}\text { Inappropriately } \\
\text { labelled }\end{array}$ & 0.4 & 4486 & 4.2 \\
\hline $\begin{array}{c}\text { Inappropriate } \\
\text { container }\end{array}$ & 0.2 & 2486 & 4.4 \\
\hline Insufficient quantity & 1.6 & 15740 & 3.7 \\
\hline Improperly stored & 1.1 & 10939 & 3.8 \\
\hline Haemolysed sample & 5.2 & 52630 & 3.2 \\
\hline Clotted sample & 1.5 & 15193 & 3.7 \\
\hline Lipemic sample & 0.1 & 1398 & 4.5 \\
\hline Contaminated sample & 0.3 & 2674 & 4.3 \\
\hline $\begin{array}{l}\text { Sample lost/not } \\
\text { received }\end{array}$ & 0.1 & 1033 & 4.6 \\
\hline $\begin{array}{l}\text { Inadequate blood } \\
\text { anticoagulant ratio }\end{array}$ & 0.2 & 1945 & 4.4 \\
\hline
\end{tabular}

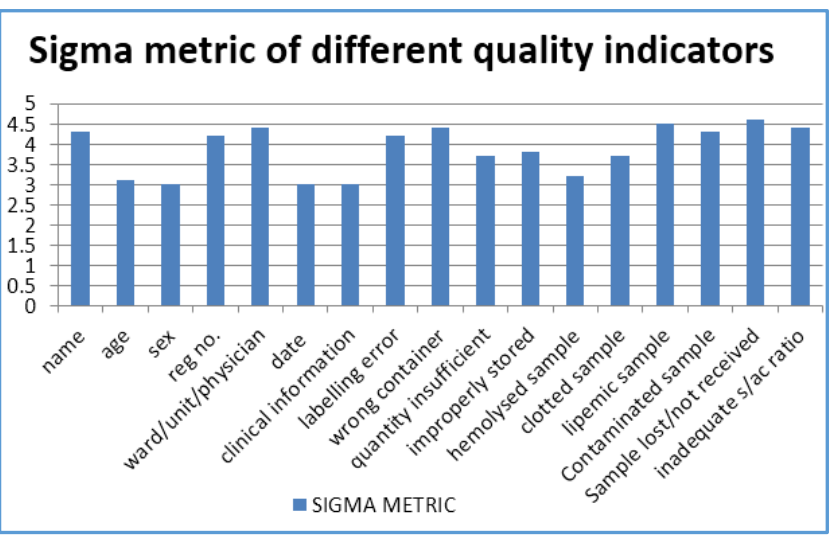

Figure 1

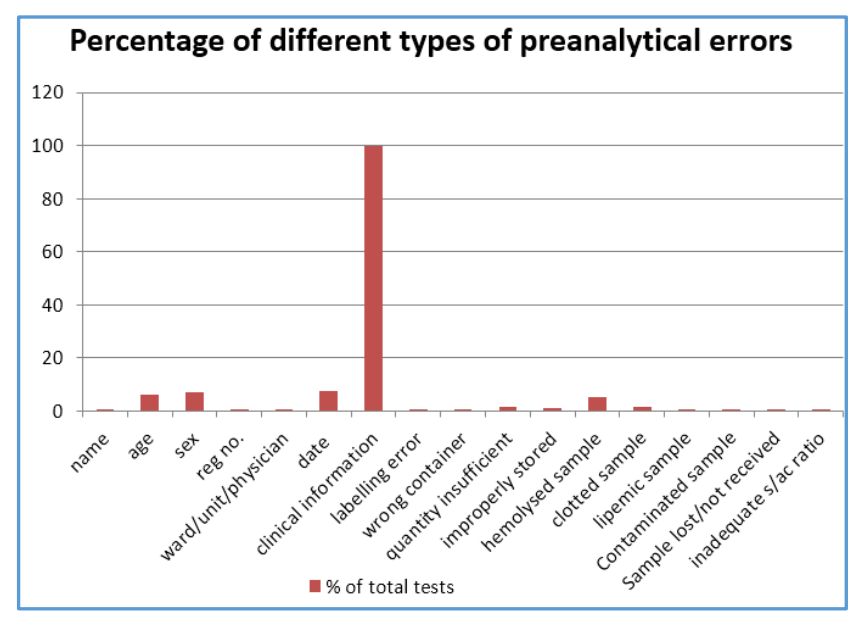

Figure. 2

\section{DISCUSSION}

In 2007, Carraro and Plebani reported $61.9 \%$ of the laboratories errors were pre-analytical, $15 \%$ were analytical, and $23.1 \%$ were post-analytical. ${ }^{10}$

Similarly, Goswami et al. found that the pre-analytical errors were the most commonly encountered, with a frequency of $77.1 \%$ followed by post analytical accounting for $15 \%$ and analytical contributing upto $7.9 \% 11$ In a survey on outpatient phlebotomy procedure, most unsuitable samples resulted from haemolysis (18.1\%), insufficient quantity (16.0\%), and clotting (13.4\%) ${ }^{12}$ These data are comparable to 
those provided by additional investigations, which confirm that problems directly related to specimen collection are the main causes of preanalytic errors, especially haemolysed, clotted, insufficient, and incorrect samples. ${ }^{13-16}$

In our study, there was a high percentage of errors in the requisition slips. $99.97 \%$ (sigma metric $<0.1$ ) of the requisition slips missed clinical information, in $7.7 \%$ (sigma metric 3) of samples, sample collection dates were missing. $7 \%$ and $6.2 \%$ samples (sigma metric 3 and 3.1 ) were without proper mention of sex and age of the patient respectively compared to $22.5 \%$ phlebotomy orders with missing information in study by Dale JC. ${ }^{12}$ This could be due to excessive patient load or lack of awareness regarding importance of patient information.

While considering errors in sample, 5.2\% samples were haemolysed (Sigma metric 3.2) while Dale JC et al found $18.1 \% .12$ Jones BA et al also found the most frequent reason for rejection was haemolysis, which occurred five times more frequently than the second most cited reason. ${ }^{14}$ Haemolysis of samples can be prevented by proper technique during blood collection, storage and sample preparation like avoiding forcing the blood through a fine needle, shaking the tubes vigorously, prolonged tourniquet application or repeated freezing and thawing of blood specimens and centrifuging after proper standing time and clot retraction.

Another factor leading to rejection of blood samples in our study was insufficient volume of blood sample and 1.6\% samples had insufficient sample quantity. The main reasons behind this may be ignorance of the phlebotomists, difficult sampling as in paediatric and geriatric patients, patients with anasarca or debilitating diseases.

In $1.5 \%$ samples, blood was clotted in spite of presence of anticoagulants. This may be due to errors in mixing the blood with anticoagulant or using vials with expiry dates.

\section{Limitations of the Study}

Further studies are necessary to bring out the differences in types of errors that may occur in samples received in our laboratory from both indoor and outdoor of COMSDH.

\section{CONCLUSION}

Effective improvements in the initial and final steps of the TTP can be achieved only if proper endeavour is taken to arrive at consensus on the preparation, adoption and monitoring of effective standard operating procedures in the initial steps of laboratory testing. Medical interns and house staffs, in spite of their theoretical knowledge, often fail to fill up requisition form properly and follow correct phlebotomy procedure. So, continuing medical education program incorporating practical skill training is extremely important. Pre-analytical errors are not inevitable. These types of errors should be monitored, documented and analysed regularly and can be avoided with a continuing education inculcating awareness, adequate laboratory personnel capable of handling a huge patient load and effective collection systems to ensure total quality patient care.

\section{REFERENCES}

[1] Lundberg GD. Acting on significant laboratory results. JAMA 1981;245(17):1762-3.

[2] Plebani M. Quality indicators to detect pre-analytical errors in laboratory testing. Clin Biochem Rev 2012;33(3):85-8.

[3] Kohn LT, Corrigan JM, Donaldson MS. To err is human: building a safer health system. Institute of Medicine (US) Committee on Quality of Health Care in America. Washington, DC: National Academies Press 2000.

[4] Plebani M, Carraro P. Mistakes in a stat laboratory: types and frequency. Clinical Chemistry 1997;43(8 Pt 1):1348-51.

[5] Baron JM, Mermel CH, Lewandrowski KB, et al. Detection of pre-analytic laboratory testing errors using a statistically guided protocol. Am J Clin Pathol 2012;138(3):406-13.

[6] Cornes MP, Atherton J, Pourmahram G, et al. Monitoring and reporting of pre-analytical errors in laboratory medicine: the UK situation. Ann Clin Biochem 2016;53(Pt 2):279-84.

[7] Sciacovelli L, Lippi G, Sumarac Z, et al. Quality indicators in laboratory medicine: the status of the progress of IFCC Working Group "Laboratory Errors and Patient Safety" project. Clin Chem Lab Med 2017;55(3):348-57.

[8] Westgard JO. Six sigma calculators. 2012.

[9] Westgard J0. Six sigma quality design and control: desirable precision and requisite $\mathrm{QC}$ for laboratory measurement processes. $2^{\text {nd }}$ edn. Madison, WI: Westgard QC Inc, 2006: p. 338.

[10] Carraro P, Plebani M. Errors in a stat laboratory: types and frequencies 10 years later. Clinical Chemistry 2007;53(7):1338-42.

[11] Goswami B, Singh B, Chawla R, et al. Evaluation of errors in a clinical laboratory: a one-year experience. Clin Chem Lab Med 2010;48(1):63-6.

[12] Dale JC, Novis DA. Outpatient phlebotomy success and reasons for specimen rejection. Arch Pathol Lab Med 2002;126(4):416-9.

[13] Bonini PA, Plebani M, Ceriotti F, et al. Errors in laboratory medicine. Clin Chem 2002;48(5):691-8.

[14] Jones BA, Calam RR, Howanitz PJ. Chemistry specimen acceptability: a College of American Pathologists QProbes study of 453 laboratories. Arch Pathol Lab Med 1997;121(1):19-26.

[15] Romero A, Munoz M, Ramos JR, et al. Identification of pre-analytical mistakes in the stat section of the clinical laboratory. Clin Chem Lab Med 2005;43(9):974-5.

[16] Jones BA, Meier F, Howanitz PJ. Complete blood count specimen acceptability. A College of American Pathologists Q-Probes study of 703 laboratories. Arch Pathol Lab Med 1995;119(3):203-8. 